\author{
K Minkner \\ K. O. Lovblad \\ H Yilmaz \\ A. Alimenti \\ L Sekoranja \\ J Delavelle \\ R Sztajzel \\ D. A. Rüfenacht
}

\section{White matter lesions in watershed territories studied with MRI and parenchymography: a comparative study}

Received: 1 June 2004

Accepted: 11 January 2005

Published online: 14 May 2005

(c) Springer-Verlag 2005
K. Minkner · K. O. Lovblad $(\bowtie)$

H. Yilmaz · A. Alimenti · J. Delavelle

D. A. Rüfenacht

Department of Radiology,

University Hospital of Geneva,

Rue Micheli-du-Crest 24,

1211 Geneva 14, Switzerland

E-mail: Karl-Olof.Lovblad@hcuge.ch

Tel.: + 41-22-3727039

Fax: + 41-22-3727072

L. Sekoranja · R. Sztajzel

Clinic of Neurology,

University Hospital of Geneva,

Rue Micheli-du-Crest 24,

1211 Geneva 14, Switzerland

\begin{abstract}
Brain aging affects an increasing segment of the population and the role of chronic cerebrovascular disease is considered to be one of the main parameters involved. For this purpose we compared retrospectively MRI data with digitized subtraction angiography (DSA) data in a group of 50 patients focusing onto the watershed area of the carotid artery vascular territories. In order to evaluate the presence of white matter lesions (WML) in the hemispheric watershed areas, coronal fluid-attenuated inversionrecovery or axial T2 weighted MRI images of patients with symptomatic cerebrovascular insufficiency areas were compared with the capillary phase of DSA studies in anteriorposterior projection. Presence of cerebrovascular occlusive disease was evaluated on DSA using North American symptomatic carotid endarterectomy trial criteria and including evaluation of collateral vascular supply. Pathological MRI findings in the region of the watershed territories correlated overall in $66 \%$ of cases with a defect or delayed filling on DSA. In the case
\end{abstract}

of asymmetrical MRI findings, there was a pathological finding of the capillary phase in the watershed area in $92 \%$ of DSA studies. Hypoperfusion in the capillary phase of the watershed area as seen on DSA correlated with the stenosis degree of the concerned carotid artery. Our findings suggest that asymmetrical findings of WML in the watershed areas as seen on MRI are caused by hemodynamic effect and a differentiation between small vessel disease and a consequence of distant stenosis may be possible under such conditions.

Keywords FLAIR - Parenchymography $\cdot$ White matter lesions . Watershed areas $\cdot$ Cerebral $\cdot$ Brain

\section{Abbreviations FLAIR:}

Fluid-attenuated inversion-recovery - WML: White matter lesions · DSP: Digitized subtraction parenchymography $\cdot$ DSA: Digitized subtraction angiography - ICA: Internal carotid artery · NASCET: North American symptomatic carotid endarterectomy trial

\section{Introduction}

A large part of the population is affected by chronic cerebrovascular disease, due to atherosclerosis of the supra-aortic vessels. Chronic cerebrovascular disease manifests itself in its terminal stages as vascular dementia, and may be associated with diffuse leucoaraiosis. Brain imaging methods that might contribute to the understanding of treatable chronic cerebrovascular disease should be sensitive to tissular changes, as well 
as capable of showing vascular occlusive disease, and be able to look specifically into the watershed area. In order to optimally select patients for treatment such as carotid endarterectomy or stenting, it is necessary to be able to assess the degree of tissular damage. On T2-weighted images and even more so on fluid-attenuated inversionrecovery (FLAIR) images, lesions due to chronic ischemia appear as confluent areas of hyperintensity in the periventricular white matter. Supplementary criteria are necessary, but a distinction between hemodynamic infarctions and lesions due to small vessel disease is complex, even though rosary like infarctions seem to be more typical in patients with hemodynamic failure due to severe carotid artery disease [1]. If combined with subcortical hyperintensity lesion and enlargement of perivascular spaces, they are more probably due to small vessel disease resulting less often in progressive stroke [2]. Periventricular hyperintensities are correlated with age and different theories about their origin are postulated [3, 4]. Stenosis of deep cerebral veins may promote development of these hyperintensities [5]. Advanced periventricular hyperintensities were more often found in patients with border-zone infarcts, and carotid atherosclerosis seems to be a predictor for border-zone infarcts [6].

The purpose of our study was to optimize the analysis of routine MRI in order to evaluate morphological data of supra aortic atheromatous disease, but also functional aspects of brain parenchyma.

\section{Materials and methods}

\section{Clinical}

A retrospective study was performed including 50 patients, who underwent both MRI and digitized subtraction angiography (DSA) for suspected cervicocranial vascular insufficiency in the carotid region. Fifty patients (37 males and 13 females) with a mean age of 70 years (range $45-87$ years) were included. Time delay between MRI and parenchymography was usually less then 4 months (mean 1.3).

\section{Inclusion criteria}

Patients had been admitted in our hospital for suspected cerebrovascular insult and had been send to radiology after clinical evaluation by a neurologist. With CT acute hemorrhage was ruled out. During their stay they were normally send to MRI. Only those patients who had also undergone angiography had been included in the study. This meant that cervical stenosis was suspected by
Doppler sonography. All patients in whom angiography revealed AVM were excluded.

\section{MRI}

In this study, we describe three regions of interest usually involved in white matter lesions: (a) the watershedterritories between the medial and anterior cerebral artery, (b) the periventricular zone (within $4 \mathrm{~mm}$ ) and the subcortical region and (c) the remaining non-a and non$b$ white matter areas.

We individualized the subgroup (a) of white matter lesions that might be the reflection of hemodynamic impairment due to atheromatous disease involving supra-aortic vessels at any level from aortic arch (ostia), carotid bifurcation, to distal internal carotid artery (ICA). The white matter lesions were defined as hyperintense lesions on FLAIR or T2 weighted sequences on MRI. Hyperintensities directly periventricular were excluded, because a probable relationship with venous congestion was proposed by Moody et al. [5]. Our study did not include the close periventricular zone within $4 \mathrm{~mm}$ and the subcortical region (b). The remaining white matter was our third group, the non-a and non-b zone (c). We described those lesions with a qualitative approach and compared them with the findings in the parenchymography (DSP).

We compared MRI T2 weighted axial or coronal FLAIR images with standardized DSP studies.

Two MR systems were used: one was an echo-planar capable $1.5 \mathrm{~T}$ system (Philips Eclipse), on which the following sequences were performed: coronal FLAIR (FSE: TE 83.2, TR 7000, TI 1700, thickness 5.0/1.0 mm, $192 \times 256$ pixels, flip angle $90^{\circ}$ ) and axial T2-weighted images (FSE: TE 96, TR 3374, thickness 5.0/1.0 mm, $256 \times 512$ pixels, flip angle $90^{\circ}$, FOV 23.0); the second system was a low-field Outlook $0.23 \mathrm{~T}$ (Philips Nordstar) on which were performed the following sequences: coronal FLAIR (IRSE: TR 3369, T1 1600, TE 120, thickness $6.0 \mathrm{~mm}$, FOV $300 \times 300 \mathrm{~mm}$, flip angle $90^{\circ}$, $162 \times 256$ pixels) and axial T2-weighted images (FSE $5900 / 80$, thickness $7.0 \mathrm{~mm}$, FOV $250 \times 270 \mathrm{~mm}$, flip angle $\left.90^{\circ}\right)$.

Digitized subtraction angiography (DSA) and digitized subtraction parenchymography (DSP)

The DSA-based parenchymography (Theron [7]; Fig. 1) was routinely performed under standard conditions during angiographic evaluation. A $40 \mathrm{cc}$ bolus of contrast material was delivered through a pigtail catheter within $2 \mathrm{~s}$ at the level of the ascending aorta. Images of the brain circulation at a frequency of two images per second were obtained in AP projection with the petrous 
Fig. 1 Technique of the digital subtraction parenchymography: bolus injection at the level of the ascending aorta and images of the brain circulation in ap projection

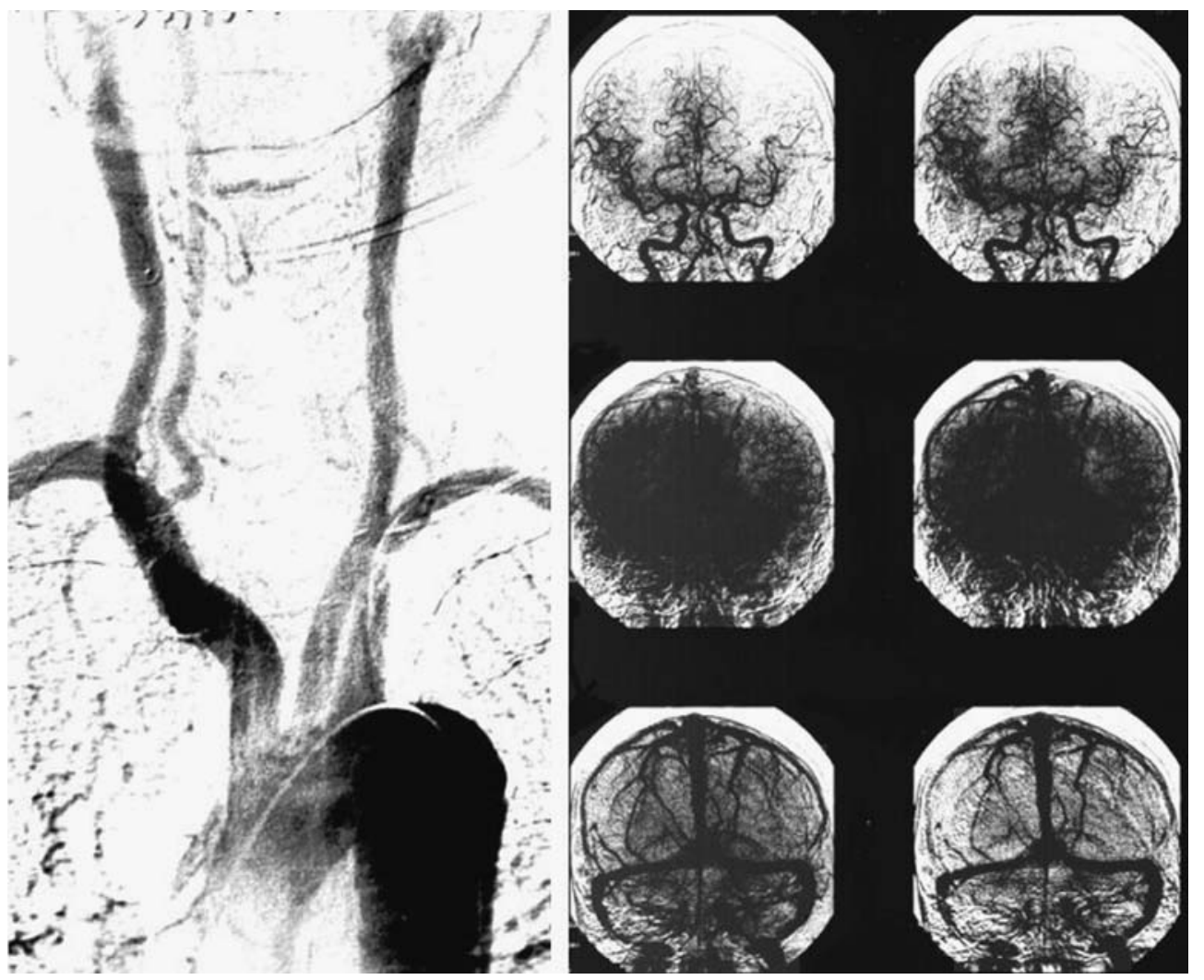

bone overlaying the lower third of the orbits. Image evaluation occurred with narrow window and low-level settings to allow visualization of the capillary stain of the brain parenchyma (DSP). The DSP provides real time information about the blood supply to the capillary bed of the watershed area (Fig. 2/Angio/Parenchymo- expl). The normal appearance of the watershed vascularization should happen synchronous on both sides [8]. We defined watershed area appearances as pathological in two situations. Either asymmetric filling (Fig. 2) of the watershed area or symmetric (Fig. 3) but delayed filling and delayed drainage of the watershed area. DSA
Fig. 2 The MRI (coronl FLAIR) and parenchymography with watershed lesions on both sides (symmetrical)

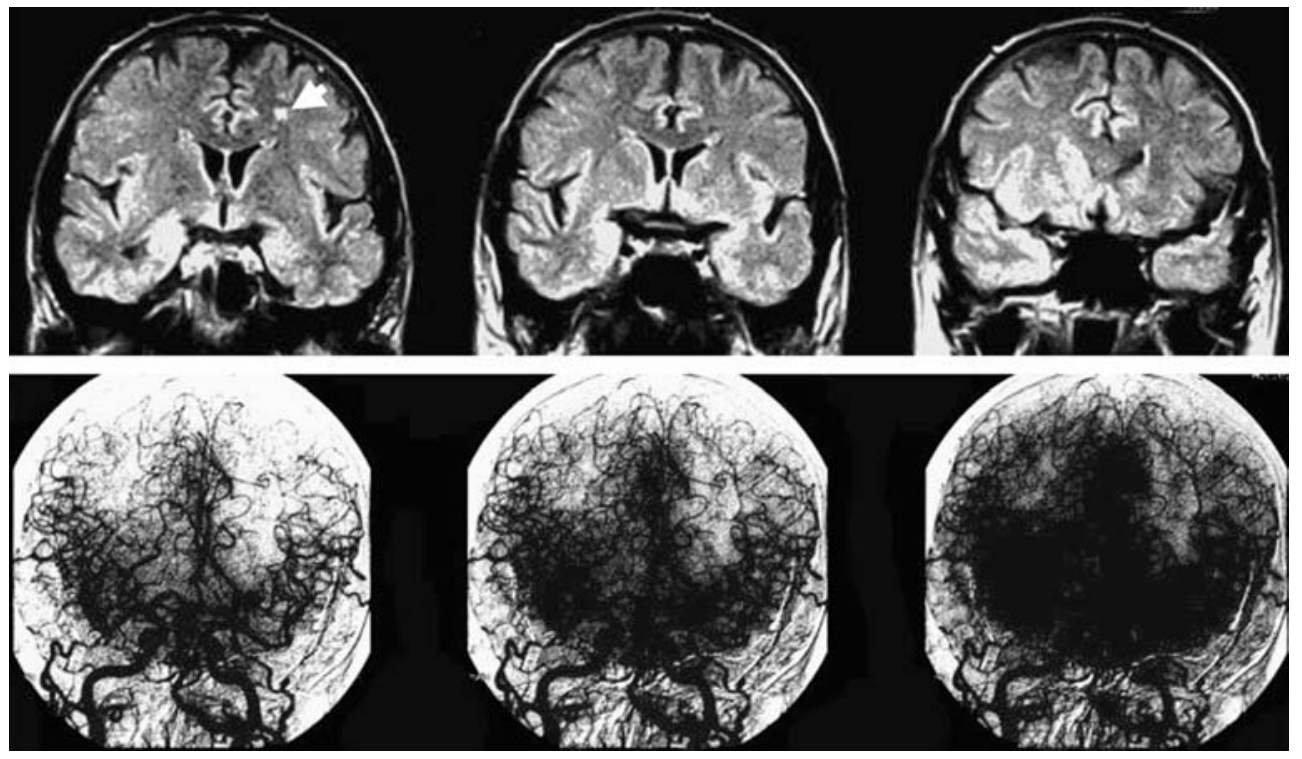


Fig. 3 The MRI (coronal $F L A I R)$ with a watershed lesion in the frontal lobe on the left side (white arrow) and parenchymography with a filling defect on the left side (asymmetrical)
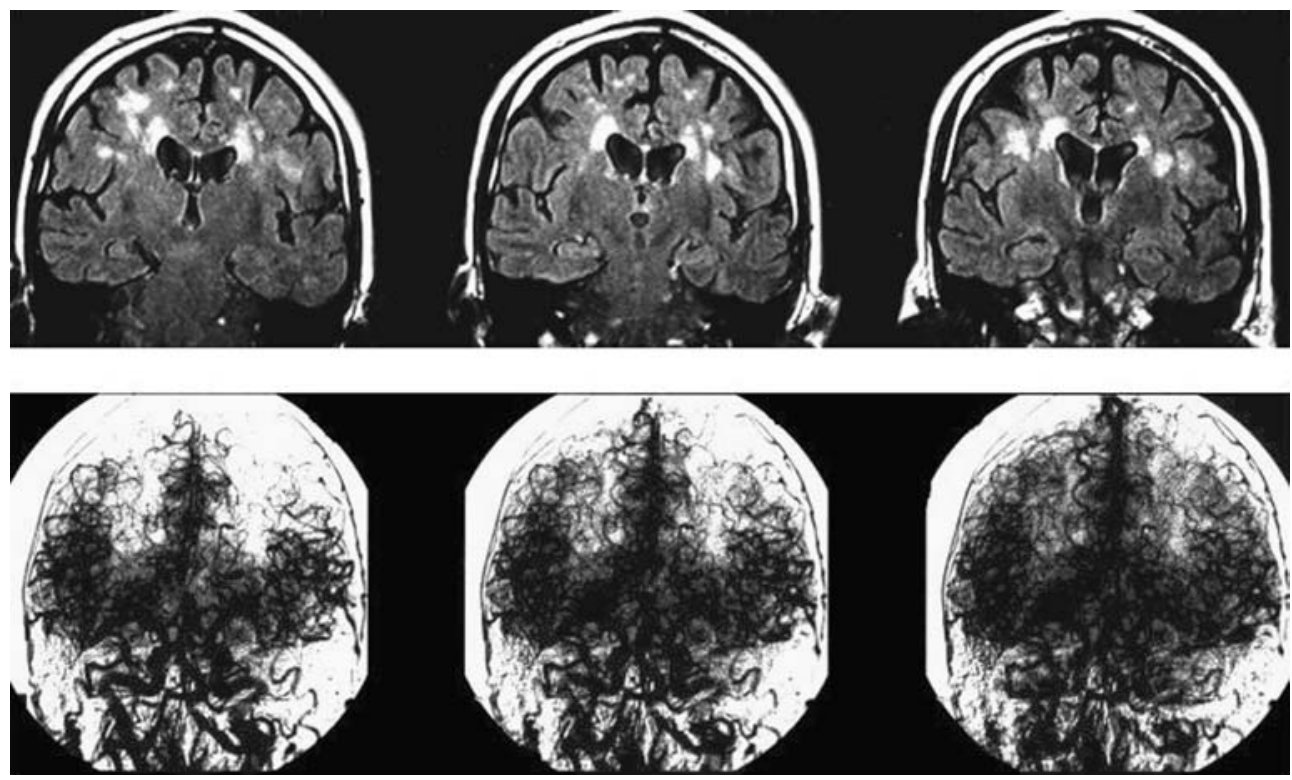

quantified carotid stenosis with North American symptomatic carotid endarterectomy trial method comparing the diameter at the site of the stenosis with the diameter of the normal artery distal to the bulb [9].

Image evaluation

Interpretation of the imaging data was organized with more than 1 year delay between hospitalizations. At that time, the reader did not know the clinical background except age and sex. One neuroradiologist performed interpretation of DSA. The degree of stenosis was given in four categories: I $<50 \%$, II $60-70 \%$, III 70-90\%, IV $>90 \%$. The MRI evaluation was performed blinded to the patient's name by the same neuroradiologist. We calculated the percentage of agreement of the lesions in MRI in region (a) compared with parenchymography.

\section{Results}

The MRI showed that 28 of 50 patients had bilateral white matter lesions of the watershed territories. Fourteen patients showed unilateral lesions and the residual eight patients showed no white matter lesions of the watershed territories (Table 1).

Of the 42 patients with either bilateral or unilateral lesions, 35 had abnormal DSP findings. Of the eight patients with no white matter lesions of the watershed territories on MRI, four showed abnormal DSP and in three, this was found associated with a unilateral stenosis. The patients with unilateral MRI findings had a good correlation with the DSP. Twelve of 14 patients (six with right and six with left sides unilateral lesions) did correlate in showing a positive finding on the corresponding side. The degree of the stenosis did show in this group a significant higher degree of stenosis on the pathologic side.

Choosing the DSP as parameter of reference we found that a defect or delayed filling in the watershed areas correlated with positive MRI findings in region (a) in $65 \%$ of the cases. Considering the patients with asymmetric MRI findings only, there was a correlation of $92 \%$. Considering the patients with symmetric pathological MRI findings only, there was a correlation of $55 \%$ (Table 2).

\section{Discussion}

In this paper, we have found evidence for a hemodynamic effect to white matter abnormalities on MRI, at least when they are found to be asymmetrical. This is consistent with previous literature; indeed, Manolio et al. [10] reported in a study of 3502 patients a strong and consistent relationship of MRI abnormalities with

Table 1 Results of DSP and MRI analyses with degree of stenosis (left/right)

\begin{tabular}{lllll}
\hline & $\begin{array}{l}\text { DSP 0/0 } \\
(n=11)\end{array}$ & $\begin{array}{l}\text { DSP 0/1 } \\
(n=18)\end{array}$ & $\begin{array}{l}\text { DSP 1/0 } \\
(n=15)\end{array}$ & $\begin{array}{l}\text { DSP 1/1 } \\
(n=6)\end{array}$ \\
\hline MRI 0/0 $(n=8)$ & $4(1.5 / 2)$ & $3(0.3 / 3.3)$ & 0 & $1(1 / 1)$ \\
MRI 0/1 $(n=7)$ & $1(0 / 0)$ & $6(1.3 / 2.3)$ & 0 & 0 \\
MRI 1/0 $(n=7)$ & $1(1 / 3)$ & 0 & $6(2.5 / 1.6)$ & 0 \\
MRI 1/1 $(n=28)$ & $5(1.4 / 1.8)$ & $9(1 / 3.2)$ & $9(3 / 1.8)$ & $5(2.6 / 3)$ \\
\hline
\end{tabular}


Table 2 Percentages of agreement of MRI with DSP

\begin{tabular}{lllll}
\hline Fifty cases 100 hemispheres & Cases & Hemispheres & DSP agreement with MRI & Disagreement with MRI \\
\hline All patients & 50 & 100 & $66 \%(n=66)$ & $34 \%(n=34)$ \\
MRI asymmetrical & 14 & 28 & $92 \%(n=26)$ & $8 \%(n=2)$ \\
MRI symmetric & 36 & 72 & $55 \%(n=40)$ & $45 \%(n=32)$ \\
DSP symmetric (Pathologic) & 6 & 12 & $83 \%(n=10)$ & $17 \%(n=2)$ \\
DSP asymmetric & 33 & 66 & $68 \%(n=45)$ & $32 \%(n=21)$ \\
DSP symmetric (normal) & 11 & 22 & $45 \%(n=10)$ & $55 \%(n=12)$ \\
\hline
\end{tabular}

carotid artery atheromatous disease. In this study, they compared carotid artery stenosis as defined by ultrasonographic techniques (found to some degree in 2745 patients) with MRI lesions (infarcts as defined by a combination of $\mathrm{T} 1$ and $\mathrm{T} 2$ signal abnormalities or FLAIR sequences) which may show areas of hyperintensities, and found that these can be related to hemodynamic impairment in correlation with the degree of carotid stenoses.

Another study reported by Adachi et al. [3] reported that there was no statistical difference between the severity of periventricular hyperintensity and extracranial arterial stenosis. Our concept of dividing the white matter in a zone near the ventricle within $4 \mathrm{~mm}$ and a more peripheral zone, which takes into account the watershed regions, explains our contradictory results compared with Adachi. The same author [2] found an association between small lacunar-like infarcts, silent subcortical hyperintensity and enlargement of the perivascular space resulting from large vessel disease. Pathological mechanisms could also depend on the precise location and its feeding artery. Read et al. [11] proposed to differentiate between white matter medullary arteries sensible for small vessel disease and the internal borderzone being the target zone for hemodynamic impairment. It remains to be proved that hyperintensities progress towards infarcts or lacunas, even if Schmidt et al. [12] showed that white matter lesions progress (in $17.9 \%$ in their study over a time period of 3 years) and cognitive impairment is associated with these lesions. Clinical progress is not yet being proved. Hund-Georgiadis et al. [13] showed that the degree of certain neurological symptoms is associated with the MR-defined severity, even though they could not confirm a significant relationship between morphological impairment and attentional deficits. Evaluating the clinical relevance and establishing additional findings is important in selecting patients for medical treatment or endarterectomy of the carotid artery.
The MRI seems to be a sensitive tool to detect watershed lesions with almost no false negative cases. On the other hand cases of no white matter lesions on MRI with significant carotid stenosis can be explained by collateral supply (circle of Willis) and hemodynamic compensation of the stenosis [14].

The rather poor specificity is probably explained by more intermittent hemodynamic factors (falls in arterial tension) in combination with vascular stenosis. Another reason is the difficulty encountered in the interpretation of parenchymography if hemodynamic impact is symmetrical. This may produce false negative results in angiography and explain the hyperintense lesions in MRI in the zone (a) with no angiographic correlation in six patients. If these are excluded from the study, MRI lesions correlate with parenchymography in $88 \%$ of the cases.

Subcortical white matter lesions at a distance of at least $4 \mathrm{~mm}$ from the ventricular surface and with a distribution corresponding to the watershed areas of the ICA territory are likely to be related to hemodynamic insufficiency of the arterial supply. Small vessel disease seems to play a much less significant role in these regions. If the watershed areas are identified and correlated with hemodynamic impacts, atherosclerosis of the larger intracranial and extracranial vessels should be looked for.

Our findings suggest that small white matter lesions in the hemispheric watershed area correlate with the presence of symptomatic cerebrovascular insufficiency. If such insufficiency is related to atheromatous stenosis of the carotid artery, the hemodynamic impact may be visualized by DSP. It may be difficult to recognize the presence of pathological changes in DSP in cases of symmetrical cerebral insufficiencies. MRI reveals watershed lesions induced by temporary perfusion changes. However, they remain invisible at DSP.

Acknowledgements Dr. Lövblad is supported by a grant from the Swiss National Science Foundation (Grant 3100-066348.01.). 


\section{References}

1. Krapf H, Widder B, Skalej M (1998) Small rosarylike infarctions in the centrum ovale suggest hemodynamic failure. Am J Neuroradiol 19:1479-1484

2. Adachi T, Kobayashi S, Yamaguchi S, Okada K (2000) MRI findings of small subcortical "lacunar-like" infarction resulting from large vessel disease. J Neurol 247:280-285

3. Adachi T, Takagi M, Hoshino H, Inafuku T (1997) Effect of extracranial carotid artery stenosis and other risk factors for stroke an periventricular hyperintensity. Stroke 28:2174-2179

4. Gawne-Cain ML, Silver NC, Moseley IF, Miller DH (1997) Fast FLAIR of the brain: the range of appearance in normal subjects and its application to quantification of white-matter disease. Neuroradiology 39:243-249

5. Moody DM, Brown RW, Challa RV, Anderson LR (1995) Periventricular venous collagenosis: association with leukoaraiosis. Radiology 194:469-476
6. Mäntylä R, Aronen HJ, Salonen $\mathrm{O}$ Pohjasvaara T, Korpelainen M, Peltonen T, Standertskjöld-Nordenstam CG Kaste M, Erkinjuntti T (1999) Magnetic resonance imaging white matter hyperintensities and mechanism of ischemic stroke. Stroke 30:2053-2058

7. Theron J, Nelson M, Alachkar F, Mazia D (1992) Dynamic digitized cerebral parenchymography. Neuroradiology 34(4):361-364

8. Théron J, Nelson M, Alachkar F, Mazia D (1992) Dynamic digitized cerebral parenchymography. Neuroradiology 34:361-364

9. North American Symptomatic Carotid Endarterectomy Trial Collaborators (1991) Beneficial effect of carotid endarterectomy in symptomatic patients with high-grade carotid stenosis. N Engl J Med 325:445-453

10. Manolio TA, Burke GL, O'Leary DH, Evans G, Beachamp N, Knepper L, Ward B (1999) Relationships of cerebral MRI findings to ultrasonographic carotid atherosclerosis in older adulds. Arterioscler Thromb Vasc Biol 19:356365
11. Read SJ, Pettigrew L, Schimmel L, Levi CR, Bladin CF, Chambers BR, Donnan GA (1998) White matter medullary infarcts: acute subcortical infarction in the centrum ovale. Cerebrovasc Dis 8:289295

12. Schmidt R, Schmidt H, Kapeller P, Lechner A, Fazekas F (2002) Evolution of white matter lesions. Cerebrovasc Dis 13(Suppl 2):16-20

13. Hund-Georgiadis M, Ballaschke O, Scheid R, Norris DG, von Cramon DY (2002) Characterization of cerebral microangiopathy using 3 T MRI: correlation with neurological impairment and vascular risk factors. J Magn Reson Imaging 15:1-7

14. Kluytmans $\mathbf{M}$, van der Grond J, van Everdingen KJ, Klijn CJM, Kappelle LJ, Viergever MA (1999) Cerebral hemodynamics in relation to patterns of collateral flow. Stroke 30:1432-1439 